\title{
Primary Amelanotic Rhabdoid Melanoma: A Case Report with Review of the Literature
}

\author{
Takahide Kaneko Ayumi Korekawa Eijiro Akasaka Daiki Rokunohe \\ Hajime Nakano Daisuke Sawamura \\ Department of Dermatology, Hirosaki Graduate School of Medicine, Hirosaki, Japan
}

\section{Key Words}

Rhabdoid melanoma $\cdot$ Malignant melanoma $\cdot$ Amelanotic melanoma $\cdot$ Phenotype

\begin{abstract}
Primary rhabdoid melanoma (PRM) is a rare variant of melanoma. Herein, we describe a case of primary amelanotic rhabdoid melanoma and review the clinicopathological features of previously reported cases of PRMs. A 63-year-old Japanese man presented with a nonpigmented red granular tumor without peripheral pigmented macules on the left heel measuring $21 \times 18 \mathrm{~mm}$ in size. Light microscopic examination revealed a tumor mass composed entirely of polygonal neoplastic cells resembling pulmonary alveoli. Tumor cells were also discohesive with bizarre nuclei, prominent nucleoli and large hyaline cytoplasmic inclusions. No melanin pigment was present. Tumor cells were strongly and diffusely positive for S-100, MART-1, HMB-45 and vimentin, while negative for desmin, aSMA and synaptophysin. According to previous reviews, PRM tends to be amelanotic and nodular. S-100 protein and vimentin stained in all cases contrary to low stainability for HMB-45, which was, by contrast, positive in our case. Prognosis of PRM remains controversial due to the very rare occurrence of this tumor and the small number of confirmed cases that have been reported. Recognition of this rare entity is important in clinical practice even for skillful dermatologists to avoid misdiagnosis with the other tumors and to determinate the subsequent treatment principles.




\section{Case Reports in Dermatology}

Kaneko et al: Primary Amelanotic Rhabdoid Melanoma: A Case Report with Review of the Literature

\section{Introduction}

Most melanomas, especially of the skin, are easily diagnosed. However, some variants have a tendency to mimic the morphology of a variety of epithelial and soft tissue neoplasms. Rhabdoid melanoma was first described by Bittesini et al. [1] in 1992, and its histological features include abundant eosinophilic cytoplasm, round vesicular nuclei with prominent, centrally placed nucleoli and large hyaline cytoplasmic inclusions.

Primary rhabdoid melanoma (PRM) is a rare variant of malignant melanoma. To the best of our knowledge, only 7 cases have been reported in the literature. Herein, we report a case of primary amelanotic rhabdoid melanoma and review the clinicopathological features of previous cases of PRMs.

\section{Case Report}

A 63-year-old Japanese man presented with a nonpigmented red granular tumor without peripheral pigmented macules on the left heel (fig. 1a). The tumor measured $21 \times 18 \mathrm{~mm}$ in size and had been detected about 1 year before. The clinical differential diagnosis included squamous cell carcinoma, poroma, porocarcinoma, metastatic skin cancer, pyogenic granuloma and amelanotic melanoma. Dermatoscopic assessment revealed a homogenous pattern with milky red areas and polymorphous vessels (fig. 1b). An incisional biopsy was performed at another institution, and a diagnosis of squamous cell carcinoma was made. He was referred to our department for treatment. CT and MRI were performed for preoperative evaluation and detected no nodal disease or distant metastasis and no other neoplasms. Hence, the patient subsequently underwent excision of the tumor with a wide margin and sentinel lymph node biopsy on the left groin.

Light microscopic examination revealed a tumor mass composed entirely of polygonal neoplastic cells predominantly resembling pulmonary alveoli with ulceration (fig. 1c). Polygonal neoplastic cells were also discohesive with bizarre nuclei, prominent nucleoli and large hyaline cytoplasmic inclusions (fig. 1d). No melanin pigment was present. An intraepidermal component was observed in multiple sections. The tumor's Breslow thickness measured $4.0 \mathrm{~mm}$, and it had a Clark level of 3 . Also, mitoses per $\mathrm{mm}^{2}$ was $>1$. Pathological examination revealed no regression and lymphovascular invasion. Infiltration of lymphocytes at the base of the tumor was mild. Similar neoplastic atypical cells were observed in the sentinel nodes, forming nests. The neoplastic cells were strongly and diffusely positive for S-100, MART-1 (fig. 2a), HMB-45 (fig. 2b) and vimentin (fig. 2c) and negative for synaptophysin, AE1/AE3 and desmin (fig. 2d).

We diagnosed this tumor as primary cutaneous amelanotic rhabdoid melanoma with characteristic features of histological examinations and immunohistochemical analysis. Clinicopathological findings resulted in a classification of stage IIIC disease in accord with the criteria set forth by the American Joint Committee on Cancer. Based on the results of the sentinel node biopsy, the patient underwent groin dissection and adjuvant combination chemotherapy that included dacarbazine. Finally, nodal involvements were found in 4 of 18 dissected nodes. At follow-up 44 months after the initial melanoma diagnosis, lung metastasis occurred. The patient has received a targeted therapy by nivolumab, which is a human programmed death receptor-1 blocking antibody at present. Incidentally, no somatic mutations in BRAF were found in this case. 
Kaneko et al.: Primary Amelanotic Rhabdoid Melanoma: A Case Report with Review of the Literature

\section{Discussion}

The typical malignant rhabdoid tumor is a pediatric malignancy occurring in the kidney, first described by Haas et al. [2] and thought to represent a rhabdomyosarcomatous variant of Wilms tumor. Presently, the malignant rhabdoid tumor is recognized as a separate entity due to a lack of rhabdomyoblastic ultrastructural features. Since first described, the presence of extrarenal malignant rhabdoid tumors has been reported in soft tissue as well as in various organs such as the brain, lung, liver, colon, ovary, uterus, and skin [3].

Rhabdoid melanoma was first described by Bittesini et al. in 1992 [1]. Since then, over 40 cases of metastatic or recurrent rhabdoid melanoma have been reported, but only 7 cases of PRM appear in the literature [4-8]. Here we report the first Japanese case of PRM in English. Clinicopathological characteristics of all PRMs are documented in table 1.

Six out of those cases showed nodular melanoma in clinical subtypes noteworthy. Moreover, 5 of these were amelanotic melanomas. Our case had both typical features of nodular melanoma and amelanotic melanoma. Furthermore, Chung et al. [8] analyzed the clinicopathological features of 31 specimens from 29 cases of metastatic rhabdoid melanoma and reported that $61 \%$ of the specimens were exclusively amelanotic and included 15 tumors with purely rhabdoid features. In both primary and metastatic cases, rhabdoid melanoma also tended to be amelanotic [9]. Although the mechanisms are still unknown, melanoma cells may lack the ability to produce melanin in the process of rhabdoid transdifferentiation.

In this case, HMB- 45 staining was clearly positive and made the diagnosis of melanoma easier. In the review of metastatic rhabdoid melanoma by Chung et al. [8], 41\% of specimens were positive for HMB-45. Low HMB-45 stainability was also found in previous PRM cases (table 1). The reasons for this phenomenon are controversial; however, we should know a lack of HMB-45 staining is a characteristic feature of rhabdoid melanoma as well as a lack of melanin pigment.

One might expect rhabdoid melanomas to follow an aggressive course because they behave like renal and extrarenal rhabdoid tumors. However, based on the previous review, rhabdoid melanomas appear to behave no more aggressively than common melanomas [3]. A confounding factor in our case is the development of lung metastasis approximately 4 years after the initial examination. Because PRMs are so rare and the number of reported cases is so small, additional research is needed to determine the prognosis of this disease with some degree of accuracy.

Rhabdoid melanoma is a rare variant of melanoma, and many pathological and biological problems have yet to be resolved. Recognition of this rare entity is important to avoid confusion both in diagnosis and in the subsequent direction of therapy.

\section{Statement of Ethics}

The authors state that the patient gave his informed consent, and it was in accordance with the guidelines approved by Hirosaki University Graduate School of Medicine.

\section{Disclosure Statement}

The authors declare no conflicts of interest. 
Kaneko et al.: Primary Amelanotic Rhabdoid Melanoma: A Case Report with Review of the Literature

\section{References}

1 Bittesini L, Dei Tos AP, Fletcher CDM: Metastatic melanoma showing rhabdoid phenotype: further evidence of a non-specific histological pattern. Histopathology 1992;20:167-170.

-2 Haas JE, Palmer NF, Weinberg AG, Beckwith JB: Ultrastructure of malignant rhabdoid tumor of the kidney. A distinct renal tumor of children. Hum Pathol 1981;12:646-657.

3 Gavino AC, Gillies EM: Metastatic rhabdoid melanoma: report of a case with a comparative review of the literature. J Cutan Pathol 2008;35:337-342.

4 Gattenlöhner S, Brocker EB, Muller-Hermelink HK: Malignant melanoma with metastatic rhabdomyosarcomartoid transdifferentiation. N Engl J Med 2008;358:649-650.

-5 Borek BT, Mckee PH, Freeman JA, Maguire B, Brander WL, Calonje E: Primary malignant melanoma with rhabdoid features: a histologic and immunocytochemical study of three cases. Am J Dermatopathol 1998;20:123-127.

-6 Parham DM, Weeks DA, Beckwith JB: The clinicopathologic spectrum of putative extrarenal rhabdoid tumors. Am J Surg Pathol 1994;18:1010-1029.

7 Tallon B, Bhawan J: Primary rhabdoid melanoma with clonal recurrence. Am J Dermatopathol 2009;31:200204.

8 Chung BY, Ahn IS, Cho SI, Kim HO, Kim KH, Park CW, Lee CH: Primary rhabdoid melanoma. Ann Dermatol 2011;23:155-159.

$>9$ Ishida M, Iwai M, Yoshida K, Kagotani A, Okabe H: Rhabdoid melanoma: a case report with review of the literature. Int J Clin Exp Pathol 2014;7:840-843.

Table 1. Clinicopathological characteristics of reported PRM

\begin{tabular}{|c|c|c|c|c|c|c|c|c|c|c|c|c|c|c|}
\hline $\begin{array}{l}\text { Case } \\
\text { [Ref.] }\end{array}$ & Age/sex & Location & $\begin{array}{l}\text { Clinical } \\
\text { subtype }\end{array}$ & $\begin{array}{l}\text { Amela- } \\
\text { notic }\end{array}$ & S-100 & MART-1 & HMB-45 & Vimentin & Desmin & $\alpha \mathrm{SMA}$ & $\begin{array}{l}\text { Synapto- } \\
\text { physin }\end{array}$ & $\begin{array}{l}\text { Follow-up } \\
\text { or survival } \\
\text { time }\end{array}$ & $\begin{array}{l}\mathrm{TT}, \\
\mathrm{mm}\end{array}$ & $\begin{array}{l}\text { Epidermal } \\
\text { components }\end{array}$ \\
\hline $1[5]$ & 36/female & Scalp & NM & yes & + & ND & - & + & - & - & ND & $12 \mathrm{mo}$ & ND & no \\
\hline $2[5]$ & 30/female & Thigh & NM & yes & + & ND & - & + & - & - & ND & $60 \mathrm{mo}$ & ND & yes \\
\hline $3[5]$ & $59 /$ male & Back & NM & yes & + & ND & - & + & - & + & ND & ND & ND & no \\
\hline $4[6]$ & 7/male & Deltoid area & ND & yes & + & ND & ND & + & ND & ND & ND & $9 \mathrm{mo}$ & ND & yes \\
\hline $5[4]$ & $41 /$ male & Scalp & ND & ND & + & + & ND & ND & ND & ND & ND & $6 \mathrm{mo},{ }^{\dagger}$ & 6 & yes \\
\hline $6[7]$ & 74/male & Back & NM & ND & + & - & - & + & ND & ND & ND & $12 \mathrm{mo}$ & ND & no \\
\hline $7[8]$ & 54/male & Forearm & NM & no & + & ND & + & + & - & - & - & $4 \mathrm{mo}$ & 9 & ND \\
\hline $\begin{array}{l}\text { Our } \\
\text { case }\end{array}$ & $63 /$ male & Sole & NM & yes & + & + & + & + & - & - & - & $44 \mathrm{mo}$ & 4 & yes \\
\hline
\end{tabular}

$\mathrm{NM}=$ Nodular melanoma; $\mathrm{ND}=$ not described or not done; $\alpha \mathrm{SMA}=$ alpha smooth muscle antigen; $\mathrm{TT}$ = tumor thickness. 


\section{Case Reports in Dermatology}
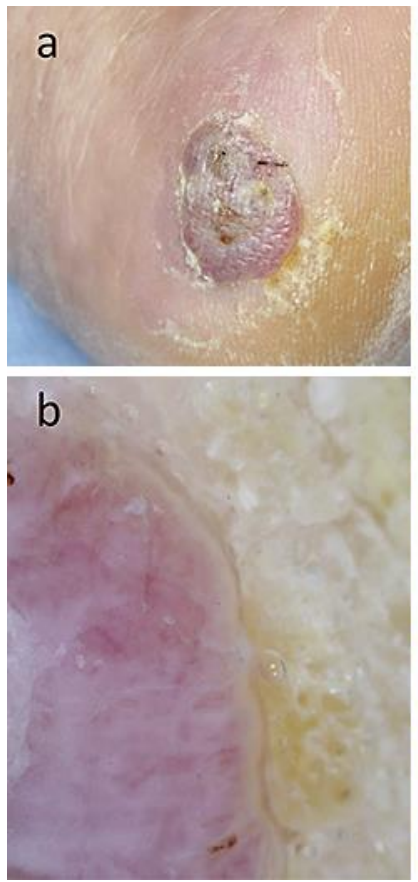

Case Rep Dermatol 2015;7:292-297

Kaneko et al.: Primary Amelanotic Rhabdoid Melanoma: A Case Report with Review of the Literature
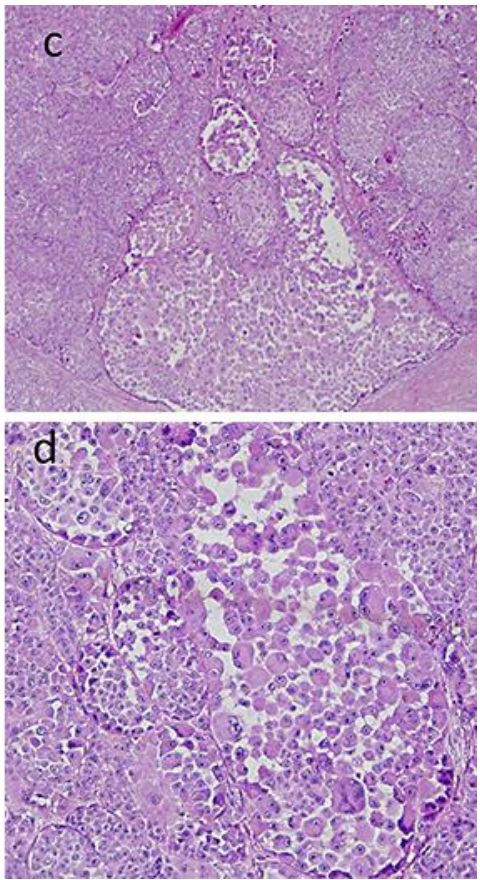

Fig. 1. a A nonpigmented red granular tumor without peripheral pigmented macules on the left heel. The tumor measured $21 \times 18 \mathrm{~mm}$ in size. b Dermatoscopy revealed a homogenous pattern with milky red areas and polymorphous vessels. c Light microscopic examination revealed a tumor mass composed entirely of polygonal neoplastic cells predominantly resembling pulmonary alveoli. d Polygonal neoplastic cells were also discohesive with bizarre nuclei, prominent nucleoli and large hyaline cytoplasmic inclusions. Hematoxylin and eosin. Original magnification. $\mathrm{c} \times 40 . \mathrm{d} \times 400$. 


\section{Case Reports in Dermatology}
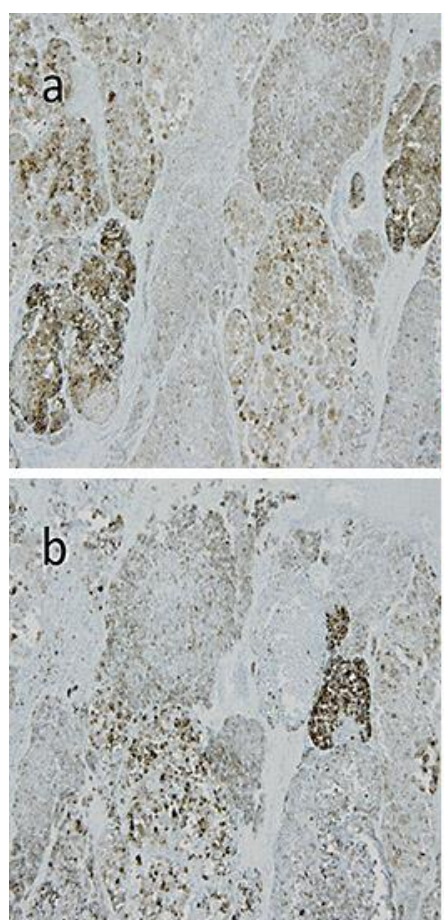

Case Rep Dermatol 2015;7:292-297

DOI: $10.1159 / 000441347$

(C) 2015 The Author(s). Published by S. Karger AG, Basel www.karger.com/cde

Kaneko et al.: Primary Amelanotic Rhabdoid Melanoma: A Case Report with Review of the Literature
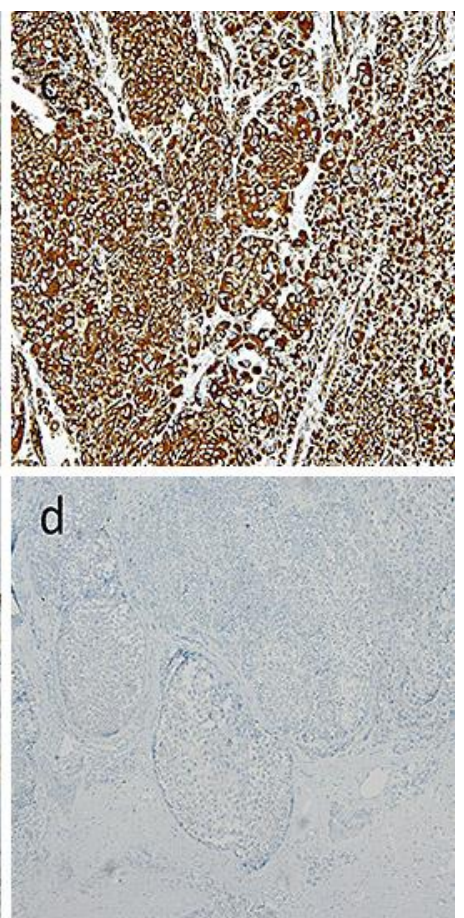

Fig. 2. Immunohistochemically, the melanoma cells were positive for MART-1 (a), HMB-45 (b) and vimentin (c), and negative for desmin (d). Original magnification. a-d $\times 40$. 